\title{
Short communication: Identification of the bovine sterol regulatory element binding protein-1c promoter and its activation by liver $X$ receptor $^{1}$
}

\author{
A. J. Lengi and B. A. Corl' \\ Department of Dairy Science, Virginia Polytechnic Institute and State University, Blacksburg 24061-0315
}

\begin{abstract}
Sterol regulatory element binding proteins (SREBP) are a family of transcription factors that regulate cholesterogenesis and lipogenesis. Sterol regulatory element binding proteins- $1 \mathrm{a}$ and $-1 \mathrm{c}$ are transcribed from the same gene by the use of alternate promoters, and only differ at their first exon. Sterol regulatory element binding protein- $1 \mathrm{c}$ is hypothesized to be an important regulator of genes involved in milk fat synthesis in the lactating dairy cow. However, the bovine SREBP-1c promoter has not been previously characterized, and studies to date that have investigated the role of SREBP-1 in the bovine mammary gland have not distinguished between isoforms 1a and 1c. The purpose of this study was to characterize the bovine SREBP-1c promoter and to investigate the DNA elements involved in the regulation of SREBP-1c expression by the liver X receptor agonist T0901317 in 2 different bovine mammary epithelial cell lines. Luciferase reporter constructs containing the wild-type SREBP-1c promoter or constructs with mutated liver $\mathrm{X}$ receptor response elements or sterol response element were transfected into MacT cells and bovine mammary epithelial (BME-UV) cells. We have demonstrated that the liver $\mathrm{X}$ receptor response elements sites in the SREBP-1c promoter are necessary for mediating the T0901317 response, and that stimulation through the sterol response element site plays only a minor role in this pathway. This report describes the bovine SREBP-1c promoter and its regulation by liver $\mathrm{X}$ receptor in bovine mammary epithelial cells.
\end{abstract}

Key words: liver X receptor, milk fat, sterol regulatory element binding protein-1

Sterol regulatory element binding proteins (SREBP) are transcription factors in the basic helix-loop-helixleucine zipper family. The SREBP are synthesized as

\footnotetext{
Received March 5, 2010.

Accepted September 1, 2010.

${ }^{1}$ This project was supported by National Research Initiative Competitive Grant No. 2009-35204-05358 from the USDA National Institute of Food and Agriculture.

${ }^{2}$ Corresponding author: bcorl@vt.edu
}

membrane-bound precursors located in the endoplasmic reticulum, which are cleaved to release their transactivation domains into the nucleus to bind to sterol response elements (SRE) in the promoters of SREBP target genes (Hua et al., 1995).

Three known SREBP isoforms are transcribed from 2 genes. Sterol regulatory element binding proteins-1a and $1 \mathrm{c}$ are produced from the same gene using alternate promoters, resulting in different first exons (exon 1a and exon 1c), both of which splice to the same second exon (Shimano, 2001). The remaining exons are common to both SREBP-1a and SREBP-1c, although reports exist of additional alternate splicing at the $3^{\prime}$ end of the human SREBP genes involving exons 18 and 19 (Eberlé et al., 2004). The alternate first exon usage leads to differences in the N-terminal transactivation domain. The shorter transactivation domain of SREBP-1c may result in this isoform being a less potent transcriptional activator than SREBP-1a (Shimano et al., 1997). The third isoform, SREBP-2, is transcribed from a separate gene (Hua et al., 1993).

Sterol regulatory element binding proteins-1a and 2 are expressed predominantly in cultured cells, whereas SREBP-1c and SREBP-2 are the predominant isoforms found in the liver and most intact organ tissues (Shimomura et al., 1997). Gene targets of SREBP are those involved in the synthesis of cholesterol, fatty acids, and triglycerides. Sterol regulatory element binding protein-1a activates transcription of all SREBP responsive genes, whereas SREBP-1c typically activates the transcription of genes involved in fatty acid synthesis, and SREBP-2 preferentially targets genes involved in cholesterogenesis (Amemiya-Kudo et al., 2002). The activity of SREBP-1a and SREBP-2 is regulated at the level of precursor protein cleavage, whereas SREBP-1c is regulated to a greater degree at the transcriptional level, in that its expression can be modulated by dietary changes (Horton et al., 1998; Eberlé et al., 2004).

The transcription of SREBP-1c has been shown to be induced by insulin in liver (Deng et al., 2002), adipose tissue, and muscle (Ducluzeau et al., 2001), and by liver $\mathrm{X}$ receptor $(\mathbf{L X R})$ activation in the liver (Repa et al., 2000). Liver X receptors are ligand-activated transcription factors in the nuclear hormone superfamily. They 
form heterodimers with retinoid $\mathrm{x}$ receptors and bind to specific DNA sequences known as LXR-response elements (LXRE) in the promoters of target genes (Fiévet and Staels, 2009). The promoter of the rodent SREBP-1c gene contains 2 LXRE and 1 SRE, which may allow for positive feedback by SREBP to increase its own transcription.

The bovine SREBP-1c promoter has not been described previously, and whereas it has been speculated that this isoform is important in the regulation of milk fat synthesis by dairy cows, its expression and regulation in bovine mammary epithelium has not been specifically examined (Harvatine and Bauman, 2006). Sterol regulatory element binding protein-1 has been reported to be highly expressed in the lactating bovine mammary gland, although the specific cell type(s) involved has not been determined. The expression level of SREBP-1 in the bovine mammary gland was decreased by treatments that cause milk fat depression, indicating that SREBP-1 is important for milk fat production (Harvatine and Bauman, 2006). Further, evidence suggests that mRNA expression of SREBP-1, in conjunction with proteins involved in the proteolytic activation of SREBP, may increase at onset of lactation in the cow (Bionaz and Loor, 2008). However, these studies did not distinguish between the SREBP-1a and SREBP-1c isoforms.

The purpose of the current study was to characterize the regulation of the bovine SREBP-1c promoter by the SRE and LXRE of the promoter in response to LXR activation in mammary epithelial cells. We identified the bovine SREBP-1c promoter by alignment with the mouse and rat SREBP-1c promoters. The sequence shown in Figure 1 was amplified by PCR from bovine genomic DNA using AccuPrime GC-Rich DNA Polymerase (Invitrogen, Carlsbad, CA) and primers as follows: F: tagcccetcgttccaccgctcttctct; R: cagatgtgggaaagccaccccettact. The resulting amplicon was cloned into the pCR4-TOPO Vector (Invitrogen), and then subcloned into the pGL4 firefly luciferase reporter vector (Promega, Madison, WI) using the SpeI restriction site in the multiple cloning site of pCR4-TOPO, and the $S m a I$ restriction site within the cloned fragment.

Transcription factor binding sites were identified within the promoter of bovine SREBP-1c using sequence homology to the well-characterized mouse, rat, and human SREBP-1c promoters, and the Pattern Search for Transcription Factor Binding Sites 1.0 search program (http://www.gene-regulation.com/cgi-bin/pub/ programs/patch/bin/patch.cgi). Identified binding elements included 2 LXRE and $1 \mathrm{SRE}$, as shown in Figure 1. These sites were destroyed by altering 3 nucleotides within each binding element using the QuikChange Lightning Site Directed Mutagenesis kit (Stratagene, La
Jolla, CA) and mutagenic primers as follows (forward orientation shown, capital letters indicated the mutated residues): mLXRE1: gctcttctctgacttgaccgccagtGaTAcctgcagacgtcg; mLXRE2: agggccagcgtccgctagtGaTAccggcccegc; mSRE: ccgcgegcgctcTcTcTatgcceggcccg. Successful mutagenesis was confirmed by sequencing.

Two different bovine mammary epithelial cell lines were examined: MacT cells and bovine mammary epithelial (BME-UV) cells. Cells were cultured at $37^{\circ} \mathrm{C}$ and $5 \% \mathrm{CO}_{2}$ in Dulbecco's modified Eagle's medium/ Ham's nutrient mixture F12 (Sigma, St. Louis, MO) supplemented with 10\% fetal bovine serum (Atlanta Biologicals, Lawrenceville, GA) and $1 \times$ antibiotic/ antimycotic solution (Sigma). Cells were trypsinized and seeded (approximately $1 \times 10^{5}$ cells per well) into 24 -well plates for $24 \mathrm{~h}$ before transfection. Medium was discarded and replaced with $1 \mathrm{~mL}$ per well of fresh growth medium or growth medium containing $10 \mu \mathrm{M}$ of the synthetic LXR agonist T0901317 (T09), $1 \mathrm{~h}$ before transfection. Cells were cotransfected with $0.5 \mu \mathrm{g}$ of the indicated pGL4 reporter construct and $30 \mathrm{ng}$ of renilla luciferase control vector (hRLuc-TK, Promega) per well, using jetPEI transfection reagent (PolyPlus Transfection, New York, NY). After $24 \mathrm{~h}$, cells were washed with PBS, lysed, and assayed for firefly and renilla luciferase activity using the Dual Luciferase Reporter Assay (Promega) and a TD-20/20 luminometer (Turner BioSystems, Sunnyvale, CA). Firefly luciferase values were normalized to renilla luciferase values for each sample. Untransfected cells and cells transfected with empty (promoterless) pGL4 were included with each experiment as negative controls (data not shown). Luminometer data were log-transformed before statistical analysis. Raw luminometer data were analyzed for normality using the "studentpanel" plot option of the MIXED procedure of SAS (v. 9.2; Cary, NC). Plots of studentized residuals and the normal probability plot were evaluated to determine that a transformation was required and that the transformation had resolved the lack of normality in the raw data. Treatments were applied as a $2 \times 4$ factorial arrangement that included 0 or $10 \mu M$ T09 and $\mathrm{WT}, \mathrm{mL} 1 \mathrm{~mL} 2, \mathrm{mS}$, or $\mathrm{mL} 1 \mathrm{~mL} 2 \mathrm{mS}$ constructs. The treatment combinations were applied in 8 replicate experiments. Data were analyzed using the MIXED procedure of SAS (v. 9.2; Cary, NC). The model accounted for the main effects of experiment, T09 inclusion, construct, and the interaction of T09 inclusion and construct. When significant interactions were detected, means were separated using Tukey's multiple comparison adjustment in the LSMeans statement. Least squares means for raw data are presented in the figures.

The MacT cells were transfected with the wildtype promoter construct or with mutated promoter 


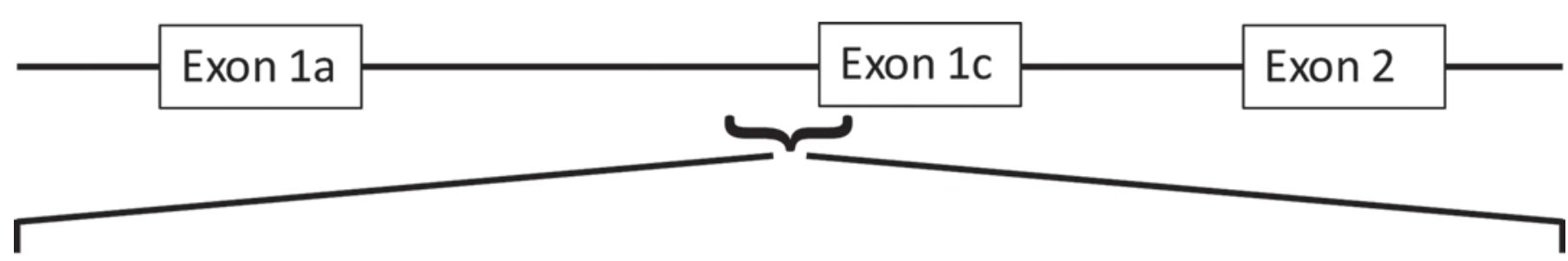

TAGCCCCTCGTTCCACCGCTCTTCTCTGACTTGACCGCCAGTAACCCCTGCAGACGTCGGCG LXRE

CCTAGTGGGTTAAGGGCCAGCGTCCGCTAGTAACCCCGGCCCCGCGGCGCAACCCGAGCC LXRE

GCGTGCCGTGGGGGTGGGGCGGGGCCGGGGCGGGGCGCCAGGAGGCGGTGGCGCGGCCGC

TGATTGGCCGCGCGCGCTCACCCCATGCCCGGCCCGCCGCTACGCGGGGCGGGGCTGGCC NF-Y SRE

GGCGGGGCGGGGCGGGGCGGGGCGGGGGCGGGGCCGGTAGCGGGGGCGGGGCCGGCGGG

TAGCTCTGTCGCTCAGCGGCTTTAAAGTGCGCCGGCTCCGCAGGTGAAGTCTGTGCTCGGAG TATA-like

ACCGGCAGGCCAGCCGCCTGGACGCCCCGAGGGGCGACGGGCCCGGGCCGCGGAACCATG Smal site

GATTGCACGTTCGAAGGTATTTTTTGGAGGCCTCCACCCCTTTATTATAGAGCTTTCATTTCCT

\section{CAGAGGGGTTCCATCCTCGGTGCCCTGAGGGGAGGTGTACACACTCCCTGCTCTAAGAGGA}

\section{GTAGGCTCCCCACGCCCACGTCCGGAGTAAGGGGGTGGCTTTCCCACATCTG}

\footnotetext{
Figure 1. Location and sequence of the PCR-amplified portion of the bovine sterol regulatory element binding protein (SREBP)-1c promoter. Notable features are underlined and include binding sites for liver X receptor response elements (LXRE), nuclear factor-Y (NF-Y), SREBP, and Sp-1, as well as the TATA-like element, the SmaI restriction site used for subcloning into the pGL4 luciferase reporter vector, and the translational start site (ATG).
}

constructs and cultured for $24 \mathrm{~h}$ with or without the synthetic LXR agonist T09 (Figure 2). In the absence of LXR activation by T09, mutations in the SRE and LXRE sites of the SREBP-1c promoter did not alter basal promoter activity. Treatment of cells with T09 resulted in a greater than 4-fold induction of the wildtype SREBP-1c promoter activity compared with basal activity $(P<0.001)$. Mutation of both LXRE sites abolished the T09 effect, in spite of the fact that any endogenous SREBP upregulated by T09 should be able to bind to the intact SRE site. This suggests that the LXRE sites are critical for mediating LXR activation of SREBP-1c in these cells, and that positive feedback from SREBP proteins binding to the SREBP-1c promoter may not play much of a role in mediating LXR activation of SREBP-1c in mammary epithelial cells.

Mutation of the SRE site in the bovine SREBP-1c promoter resulted in significantly reduced T09-induced promoter activity compared with the wild-type promoter $(P<0.01)$. However, the T09 effect was still significant, with a 2.6-fold increase in luciferase activity, compared with unstimulated MacT cells transfected with the same mutated construct $(P<0.001)$. Together, these results suggest that whereas the SRE site might 


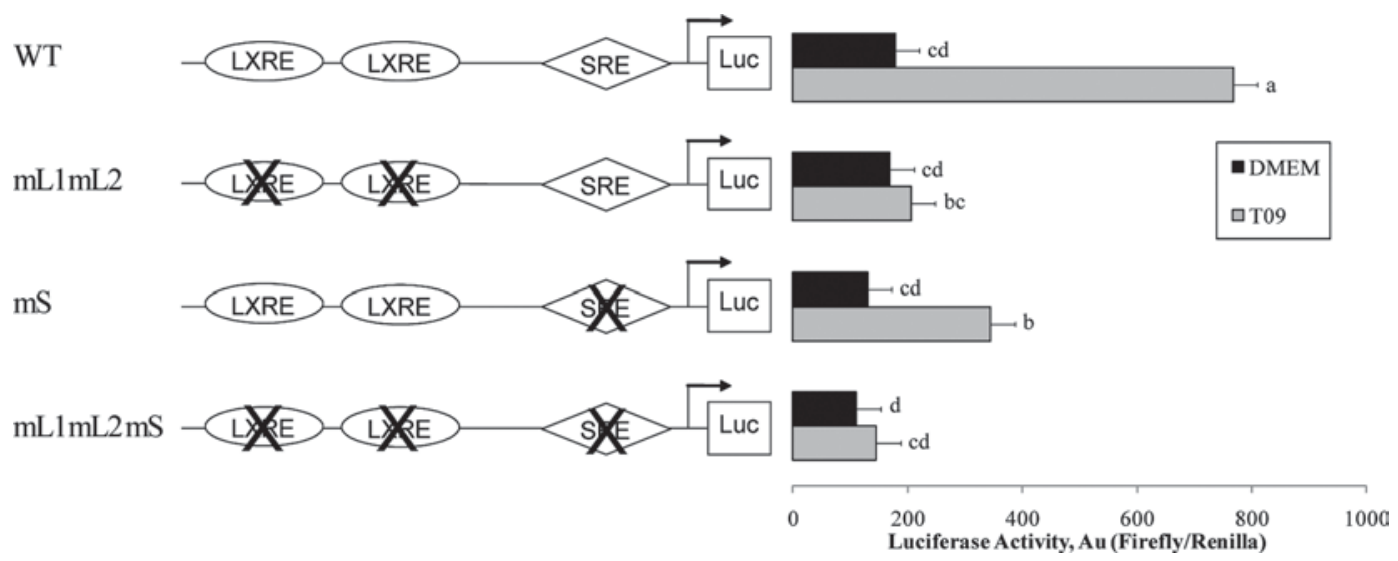

Figure 2. Mutations in the bovine sterol regulatory element binding protein-1c promoter suppress transcriptional stimulation in response to the synthetic LXR agonist T0901317 (T09) in MacT cells. Cells were co-transfected with either wild-type or mutated promoter constructs, as indicated, and a renilla luciferase reference vector, and then cultured with or without $10 \mu M$ T09 for 24 h. Firefly luciferase activity was measured and normalized to renilla luciferase activity for each sample. Results are presented as the means of 8 experiments $(\mathrm{n}=8)+$ SEM; ${ }^{\mathrm{a}-\mathrm{d}}$ means lacking a common letter are different $(P<0.05)$. DMEM $=$ Dulbecco's modified Eagle's medium; LXRE $=$ liver X receptor response elements; $\mathrm{SRE}=$ sterol response elements; $\mathrm{Au}=$ arbitrary units; WT = wild type promoter; $\mathrm{mL} 1 \mathrm{~mL} 2=$ mutated LXRE; $\mathrm{mS}=$ mutated SRE; $\mathrm{mL} 1 \mathrm{~mL} 2 \mathrm{mS}=$ mutated LXRE and SRE.

not be necessary for LXR activation of the SREBP1c promoter, it may play a role in maximal promoter activation. Mutation of all 3 sites abolished the T09 effect, and promoter activity in cells transfected with this construct was indistinguishable from basal activity. Similar findings were made in a study of rat SREBP-1c regulation using primary rat hepatocytes, in which mutation of both LXRE sites resulted in an $84 \%$ reduction in T09-induced promoter activation, whereas mutation of the SRE site had a minor effect on stimulation of the promoter by T09 (Cagen et al., 2005). Our results are also in agreement with similar studies performed in HEK-293 cells using the human SREBP-1c promoter, which differs from the mouse, rat, and bovine SREBP1c promoter in that it contains 2 SRE sites rather than 1 (Dif et al., 2006). In this study, mutation of both LXRE sites abolished T09-induced promoter activity, despite the fact that 2 SRE sites remained intact.

We repeated the experiment using a second bovine mammary epithelial cell line, BME-UV cells. Previous work in our laboratory has shown that culturing BMEUV cells with $2 \mu M$ T09 results in increased lipogenesis (as measured by radiolabeled acetate incorporation) as well as increased mRNA and protein expression of SREBP1, although no distinction was made between the 1a and 1c isoforms (McFadden and Corl, 2010). In the present study, BME-UV cells showed a similar pattern to the results obtained with MacT cells (Figure 3). Mutation of the SRE and LXRE did not alter basal promoter activity; however, treatment of BME-UV cells with T09 resulted in a significant (greater than 4-fold) induction of the wild-type SREBP-1c promoter activity compared with basal wild-type promoter activity $(P$
$<0.001$ ), and mutation of both LXRE sites abolished the T09 effect. Unlike the MacT cells, mutation of the SRE site in these cells did not significantly reduce T09induced promoter activity compared with the wild-type promoter. Treatment of BME-UV cells transfected with the mSRE promoter construct with T09 resulted in a significant increase in luciferase activity above unstimulated BME-UV cells transfected with the same mutated construct $(P<0.01)$. Mutation of all 3 sites abolished the T09 effect, and promoter activity in cells transfected with this construct was indistinguishable from basal activity.

Whereas the SRE and LXRE sites appear to be important for mediating T09-induced activation, the SREBP-1c promoter also contains several other transcription factor binding sites that may be important for basal activation, or activation through other pathways. In the rodent SREBP-1c promoter, the SRE site is found within a region of DNA termed the "SRE complex," which contains several other consensus transcription factor binding sites, including a site for nuclear factor-Y, an E-box, and an Sp-1 site (Amemiya-Kudo et al., 2000). Co-factor binding of these sites has been shown to play a role in the regulation of other SREBPresponsive genes, such as spot14 (Jump et al., 2001), fatty acid synthase (Magaña et al., 2000), and adenosine triphosphate-citrate lyase (Moon et al., 2000). In HEK-293 cells transfected with murine SREBP-1c promoter luciferase reporter constructs, all of these sites were shown to be necessary for maximal stimulation of the SREBP-1c promoter in response to $10 \mu M$ pravastatin (Amemiya-Kudo et al., 2000). In contrast, a similar study using rat hepatocytes transfected with rat 


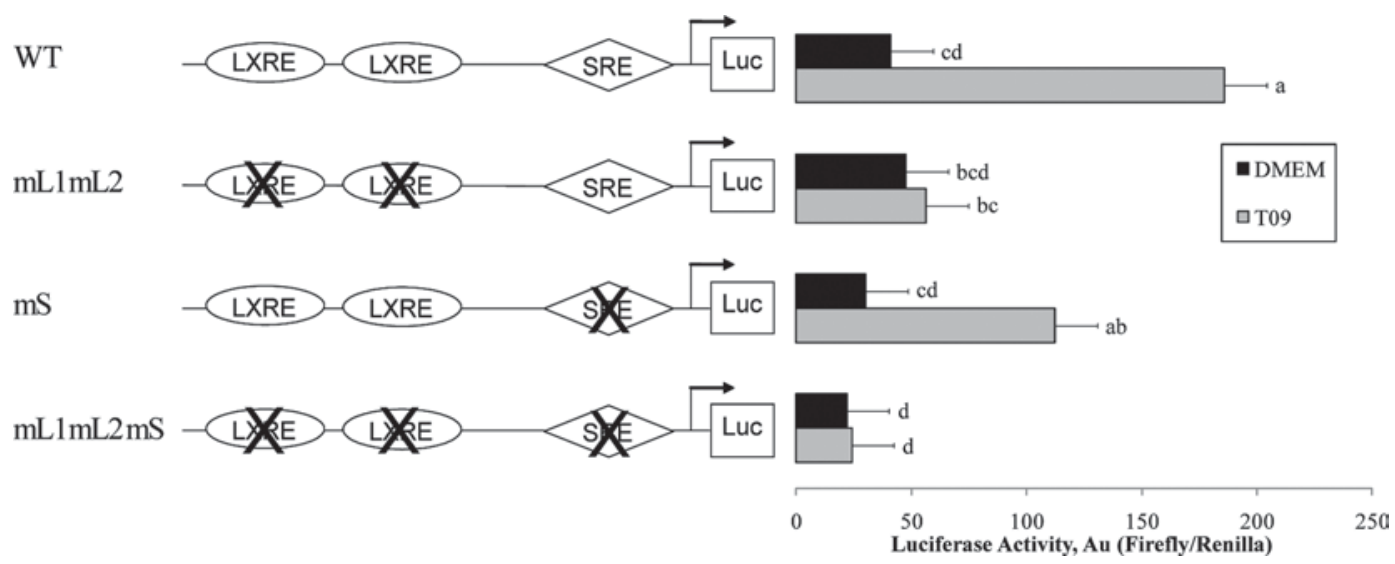

Figure 3. Mutations in the bovine sterol regulatory element binding protein-1c promoter suppress transcriptional stimulation in response to the synthetic LXR agonist T0901317 (T09) in bovine mammary epithelial (BME-UV) cells. Cells were co-transfected with either wild-type or mutated promoter constructs, as indicated, and a renilla luciferase reference vector, and then cultured with or without $10 \mu M \mathrm{~T} 09$ for $24 \mathrm{~h}$. Firefly luciferase activity was measured and normalized to renilla luciferase activity for each sample. Results are presented as means of 8 experiments $(\mathrm{n}=8)+$ SEM; ${ }^{\text {a-d }}$ means lacking a common letter are different $(P<0.05)$. DMEM $=$ Dulbecco's modified Eagle's medium; LXRE $=$ liver $\mathrm{X}$ receptor response elements; $\mathrm{SRE}=$ sterol response elements; $\mathrm{Au}=$ arbitrary units.

SREBP-1c promoter luciferase constructs showed that mutation of the Sp-1 site reduced promoter stimulation by T09, but mutation of the E-box site had no effect (Cagen et al., 2005). The human SREBP-1c promoter appears to differ from the rodent promoter in that it contains 2 SRE sites but no consensus E-box (Dif et al., 2006). The bovine SREBP-1c promoter differs from both rodents and humans; it does not appear to contain a consensus E-box and has just 1 SRE site, although nuclear factor-Y and Sp-1 sites are present. It is unclear what role these sites might play in the regulation of SREBP-1c in bovine mammary epithelium.

Sterol regulatory element binding proteins are thought to be critical transcription factors regulating the genes involved in milk fat synthesis in the lactating dairy cow (Peterson et al., 2004). Because of the role that SREBP-1c plays in lipogenesis and triglyceride synthesis in other tissues, it has been hypothesized to be the predominant isoform expressed in mammary tissue (Harvatine and Bauman, 2006). However, bovine SREBP-1c has not been previously studied. Relatively little is known about the role of SREBP-1c in the mammary gland during lactation; many of the initial studies examining the regulation of SREBP-1c were performed with primary hepatocytes or hepatoma cell lines. One study of the murine mammary gland found that the mRNA expression of SREBP-1c increased 1.76-fold at the onset of lactation, whereas the expression of the other SREBP isoforms remained constant. This increase, however, was not statistically significant (Rudolph et al., 2007). Currently available studies of SREBP expression and regulation in bovine mammary tissue do not distinguish between SREBP-1a and SREBP-1c (Peterson et al., 2004; Harvatine and Bauman, 2006; Bionaz and Loor, 2008).

In conclusion, we have shown that activating LXR using a synthetic ligand resulted in increased promoter activity of SREBP-1c in both bovine mammary epithelial cell lines examined. The proximal promoter of bovine SREBP-1c includes binding sites for LXR and SREBP, and the presence of a SRE in the promoter of SREBP-1c could allow for positive feedback regulation. Mutation of the LXR binding sites in the promoter eliminated the stimulating effect of LXR activation despite the potential for activation of endogenous SREBP-1 by LXR followed by binding to the promoter SRE. Mutation of the SRE reduced the promoter activation by LXR, but stimulation was still greater than basal activity indicating that LXR alone is capable of stimulating SREBP-1c promoter activity, but that SREBP binding to the promoter is required for maximal stimulation. To our knowledge, this is the first study examining the SREBP-1c promoter in bovine mammary epithelial cells.

\section{REFERENCES}

Amemiya-Kudo, M., H. Shimano, A. H. Hasty, N. Yahagi, T. Yoshikawa, T. Matsuzaka, H. Okazaki, Y. Tamura, Y. Iizuka, K. Ohashi, J. Osuga, K. Harada, T. Gotoda, R. Sato, S. Kimura, S. Ishibashi, and N. Yamada. 2002. Transcriptional activities of nuclear SREBP-1a, -1c, and -2 to different target promoters of lipogenic and cholesterogenic genes. J. Lipid Res. 43:1220-1235.

Amemiya-Kudo, M., H. Shimano, T. Yoshikawa, N. Yahagi, A. H Hasty, H. Okazaki, Y. Tamura, F. Shionoiri, Y. Iizuka, K. Ohashi, J. Osuga, K. Harada, T. Gotoda, R. Sato, S. Kimura, S. Ishibashi, and N. Yamada. 2000. Promoter analysis of the mouse sterol regulatory element-binding protein-1c gene. J. Biol. Chem. 275:31078-31085. 
Bionaz, M., and J. J. Loor. 2008. Gene networks driving bovine milk fat synthesis during the lactation cycle. BMC Genomics 9:366.

Cagen, L. M., X. Deng, H. G. Wilcox, E. A. Park, R. Raghow, and M. B. Elam. 2005. Insulin activates the rat sterol-regulatory-elementbinding protein 1c (SREBP-1c) promoter through the combinatorial actions of SREBP, LXR, Sp-1 and NF-Y cis-acting elements. Biochem. J. 385:207-216.

Deng, X., L. M. Cagen, H. G. Wilcox, E. A. Park, R. Raghow, and M. B. Elam. 2002. Regulation of the rat SREBP-1c promoter in primary rat hepatocytes. Biochem. Biophys. Res. Commun. 290:256-262.

Dif, N., V. Euthine, E. Gonnet, M. Laville, H. Vidal, and E. Lefai. 2006. Insulin activates human sterol-regulatory-element-binding protein-1c (SREBP-1c) promoter through SRE motifs. Biochem. J. 400:179-188.

Ducluzeau, P. H., N. Perretti, M. Laville, F. Andreelli, N. Vega, J. P. Riou, and H. Vidal. 2001. Regulation by insulin of gene expression in human skeletal muscle and adipose tissue. Evidence for specific defects in type 2 diabetes. Diabetes 50:1134-1142.

Eberlé, D., B. Hegarty, P. Bossard, P. Ferre, and F. Foufelle. 2004 SREBP transcription factors: Master regulators of lipid homeostasis. Biochimie 86:839-848.

Fiévet, C., and B. Staels. 2009. Liver X receptor modulators: Effects on lipid metabolism and potential use in the treatment of atherosclerosis. Biochem. Pharmacol. 77:1316-1327.

Harvatine, K. J., and D. E. Bauman. 2006. SREBP1 and thyroid hormone responsive spot 14 (S14) are involved in the regulation of bovine mammary lipid synthesis during diet-induced milk fat depression and treatment with CLA. J. Nutr. 136:2468-2474.

Horton, J. D., Y. Bashmakov, I. Shimomura, and H. Shimano. 1998. Regulation of sterol regulatory element binding proteins in livers of fasted and refed mice. Proc. Natl. Acad. Sci. USA 95:59875992

Hua, X., J. Wu, J. L. Goldstein, M. S. Brown, and H. H. Hobbs. 1995. Structure of the human gene encoding sterol regulatory element binding protein-1 (SREBF1) and localization of SREBF1 and SREBF2 to chromosomes $17 \mathrm{p} 11.2$ and 22q13. Genomics 25:667-673.

Hua, X., C. Yokoyama, J. Wu, M. R. Briggs, M. S. Brown, J. L. Goldstein, and X. Wang. 1993. SREBP-2, a second basic-helixloop-helix-leucine zipper protein that stimulates transcription by binding to a sterol regulatory element. Proc. Natl. Acad. Sci. USA 90:11603-11607.

Jump, D. B., A. P. Thelen, and M. K. Mater. 2001. Functional interaction between sterol regulatory element-binding protein-1c, nuclear factor Y, and 3,5,3'-triiodothyronine nuclear receptors. J. Biol. Chem. 276:34419-34427.

Magaña, M. M., S. H. Koo, H. C. Towle, and T. F. Osborne. 2000. Different sterol regulatory element-binding protein-1 isoforms utilize distinct co-regulatory factors to activate the promoter for fatty acid synthase. J. Biol. Chem. 275:4726-4733.

McFadden, J. W., and B. A. Corl. 2010. Activation of liver X receptor (LXR) enhances de novo fatty acid synthesis in bovine mammary epithelial cells. J. Dairy Sci. 93:4651-4658.

Moon, Y. A., J. J. Lee, S. W. Park, Y. H. Ahn, and K. S. Kim. 2000. The roles of sterol regulatory element-binding proteins in the transactivation of the rat ATP citrate-lyase promoter. J. Biol. Chem. 275:30280-30286.

Peterson, D. G., E. A. Matitashvili, and D. E. Bauman. 2004. The inhibitory effect of trans-10, cis-12 CLA on lipid synthesis in bovine mammary epithelial cells involves reduced proteolytic activation of the transcription factor SREBP-1. J. Nutr. 134:2523-2527.

Repa, J. J., G. Liang, J. Ou, Y. Bashmakov, J. M. Lobaccaro, I. Shimomura, B. Shan, M. S. Brown, J. L. Goldstein, and D. J. Mangelsdorf. 2000. Regulation of mouse sterol regulatory element-binding protein-1c gene (SREBP-1c) by oxysterol receptors, LXRalpha and LXRbeta. Genes Dev. 14:2819-2830.

Rudolph, M. C., J. L. McManaman, T. Phang, T. Russell, D. J. Kominsky, N. J. Serkova, T. Stein, S. M. Anderson, and M. C. Neville. 2007. Metabolic regulation in the lactating mammary gland: A lipid synthesizing machine. Physiol. Genomics 28:323-336.

Shimano, H. 2001. Sterol regulatory element-binding proteins (SREBPs): Transcriptional regulators of lipid synthetic genes. Prog. Lipid Res. 40:439-452.

Shimano, H., J. D. Horton, I. Shimomura, R. E. Hammer, M. S. Brown, and J. L. Goldstein. 1997. Isoform 1c of sterol regulatory element binding protein is less active than isoform 1a in livers of transgenic mice and in cultured cells. J. Clin. Invest. 99:846-854.

Shimomura, I., H. Shimano, J. D. Horton, J. L. Goldstein, and M. S. Brown. 1997. Differential expression of exons 1a and 1c in $\mathrm{mR}-$ NAs for sterol regulatory element binding protein-1 in human and mouse organs and cultured cells. J. Clin. Invest. 99:838-845. 